\title{
Jahrestagung 1979
}

Zum zweiten Male seit ihrer Wiedergründung 1949 kehrte die Vereinigung an ihren Gründungsort von 1922 zurück: nach Berlin. Sie wählte diese Stätte im Jubiläumsjahr 1979 mit gutem Grund und mit deutlichem Bezug zum ersten Beratungsgegenstand. Fast 200 Mitglieder mit ihren Damen sowie mehrere der Vereinigung seit längerem verbundene Fachkollegen aus dem Ausland und die Schriftleiter der juristischen Fachpresse besuchten die Tagung und die Veranstaltungen der Vereinigung.

In einem von dem Herrn Präsidenten des Deutschen Bundestages dankenswerterweise zur Verfügung gestellten Saal des Reichstagsgebäudes konnte der Erste Vorsitzende der Vereinigung, Klaus Stern (Köln), zu den Referaten und der Diskussion des ersten Beratungsgegenstandes auch das Mitglied der Vereinigung, Herrn Bundespräsidenten Professor Dr. Karl Carstens, begrüßen.

Die Diskussionsleitung am ersten Tage lag in der Hand des Zweiten Vorsitzenden, Thomas Oppermann (Tübingen). Die Verhandlungen zum zweiten Beratungsgegenstand leitete der Dritte Vorsitzende, Rupert Scholz (München).

In der Mitgliederversammlung wurde der verstorbenen Kollegen Richard Naumann (Hamburg/Lüneburg), Christoph Sasse (Hamburg), Eberhard Menzel (Kiel), Walter Hamel (Marburg) gedacht. Außerdem wurden Satzungsfragen und Regularien behandelt sowie $15 \mathrm{neu}$ aufgenommene Mitglieder vorgestellt. Als neuer Vorstand wurde in geheimer Wahl gewählt: Günther Winkler (Wien), Martin Bullinger (Freiburg), Wolfgang Martens (Hamburg).

Die Jahrestagung 1980 wurde vom 1. bis 4. Oktober nach Innsbruck vergeben.

Die Arbeitssitzungen der Vereinigung wurden von Empfängen umrahmt: Am ersten Abend lud der Vizepräsident der Freien Universität, Herr Professor Dr. Dieter Heckelmann, in den Henry-Ford-Bau ein, am zweiten Tag Herr Bürgermeister Wolfgang Lüder in die Eichengalerie des Schlosses Charlottenburg. Die wissenschaftliche Tagung beendete der schon fast traditionelle festliche Gesellschaftsabend, der diesmal im Schloßhotel Gehrhus stattfand. Am Samstag konnte Herr Senatsdirektor Professor Dr. Hartmut Jäckel die Vereinigung auf dem Motorschiff „Großer Kurfürst" zu einer Rundfahrt auf der 
Havel begrüßen, die bei strahlendem Sonnenschein Berlins landschaftliche Schönheit ebenso vor Augen führte wie seine schreckliche Teilung durch Mauer und Stacheldraht.

Besonderen Dank schuldet die Vereinigung dem Berliner Organisationskomitee, allen voran dem kooptierten Vorstandsmitglied Dieter Wilke und seiner Gattin sowie allen Mitarbeiterinnen und Mitarbeitern der Berliner Fakultät, die keine Mühe scheuten, der Tagung einen reibungslosen Ablauf zu sichern.

Die nachstehend abgedruckten Referate wurden jeweils am Vormittag des 4. und 5. Oktober gehalten. Ihnen folgte am Nachmittag die vollständig wiedergegebene Diskussion. 